\title{
Uncertainty visualization using maps for nuclear and radiological emergencies
}

\author{
A. Nagy ${ }^{1, *}$, T. Perko ${ }^{1}$, T. Müller ${ }^{2}$, W. Raskob ${ }^{2}$ and L. Benighaus ${ }^{3}$ \\ 1 SCK-CEN-Belgian Nuclear Research Centre, Mol, Belgium. \\ ${ }^{2}$ KIT - Karlsruhe Institute of Technology, Eggenstein-Leopoldshafen, Germany. \\ ${ }^{3}$ DIALOGIK - non-profit institute for communication and cooperation research, Stuttgart, Germany.
}

\begin{abstract}
Visual uncertainty representation and communication during nuclear and radiological emergencies face empirical and theoretical challenges. The work carried out focuses on visualisation of uncertainties as a decision support and communication tool. We represent uncertainty visually through maps and visual robustness indicators. An interdisciplinary approach was applied where both quantitative and qualitative methods were used to validate and test the tools developed in 6 different occasions/countries. The principles for building effective uncertainty representation and communication tools are presented. It is important to note that the visual approach offers a great opportunity to represent uncertainty to experts in the field of emergency planning especially in the nuclear/radiological one.
\end{abstract}

Keywords: nuclear / radiological / emergency / maps / robustness indicators / human computer interaction / uncertainty / visualisation / communication / knowledge modelling

\section{Introduction}

The way we visualize information related to radiological releases in emergency situations and associated uncertainties is of extreme importance for decision-making, both for experts involved in emergency management, as well as for the potentially affected population. Our current work builds on the results of PREPARE project especially on the work carried out for 1) information representation and 2) communication with the public (Marignac et al., 2016; Raskob et al., 2016). We extend the work further in two innovative directions: 1) we represent uncertainty visually and 2) integrate the tools in the decision-making cycle. As a common practice, experts produce a series of maps (e.g., ongoing or predicted releases, doses to the population, radioactive contamination, affected areas). They use different software tools and standards (e.g., specialized systems such as RODOS, GIS and Google maps) and apply different visualizations (e.g., colors, contours and measurement units). Decision-makers at different levels, from federal level to the mayor of a local community, and first responders need to interpret these maps and advise the population on protective behavior (e.g., consumption advice of vegetables from gardens of an affected area). Mass media often refer to these maps and publish them on-line (e.g., interactive maps). Maps also appear in social media (e.g., blogs and

\footnotetext{
*Corresponding author: ahmed.nagy@sckcen. be
}

tweets). Affected population need to understand maps to know when to take certain actions in case of an emergency, and what to avoid (e.g., which roads to take during an evacuation, at what time).

A visual representation of the data conveys the uncertainty associated with the information used to derive decisions. However, conveying uncertainty only might increase the challenges a decision maker has to face in order to reach a well-informed robust decision. Pang defined uncertainty by presenting three types that can impact the comprehension and the decisions based on the data: 1) data uncertainty; 2) derived uncertainty and 3) visual uncertainty (Pang et al., 1997). Further, Pang introduced a concise taxonomy for visualization methods of uncertainty using glyphs including contour lines and pseudo coloring techniques (Pang et al., 1997). In another scholar work, Hunter defined uncertainty as the "degree to which the lack of knowledge about the amount of error is responsible for hesitancy in accepting results and observations with caution" (Hunter and Goodchild, 1993). Thomson developed a taxonomy of uncertainty relevant to visualization (Thomson et al., 2005), by defining core aspects of uncertainty as: accuracy/error, precision, completeness, consistency, lineage (provenance), subjectivity and interrelatedness.

Work in uncertainty can be categorized as uncertainty modelling and uncertainty propagation. This article focusses on visualisation of uncertainties on maps. It identifies main misinterpretations of radiation dispersion maps used for nuclear or radiological emergencies. We present maps and 
Table 1. Summary of the methods used for testing the tools.

\begin{tabular}{|c|c|c|c|}
\hline Country/Event & $\begin{array}{l}\text { Method for input } \\
\text { collection }\end{array}$ & $\begin{array}{l}\text { Number of } \\
\text { participants }\end{array}$ & Participants \\
\hline Slovak Republic & Workshop & 21 & $\begin{array}{l}\text { Decision-makers involved at different levels of the emergency } \\
\text { preparedness, response and recovery management activities. }\end{array}$ \\
\hline NERIS workshop 2019 & Workshop & 19 & Experts and decision-makers from various countries \\
\hline Spain & Interviews & 3 & $\begin{array}{l}\text { Expert decision-makers from different emergency planning and } \\
\text { response organisations }\end{array}$ \\
\hline Norway/Sweden & Discussions during lectures & $15+23$ & $\begin{array}{l}\text { Students of the course on radiation in the environment and NEA/ } \\
\text { SU Radiation Protection course }\end{array}$ \\
\hline $\begin{array}{l}\text { RICOMET } 2018 \\
\text { conference }\end{array}$ & Discussion after presentation & 53 & Experts with different tasks in emergency management \\
\hline
\end{tabular}

robustness indicators as uncertainty communication and representation tool. We further share our recommendations on how to limit the introduction of new uncertainties for visual uncertainty representations.

The following section describes methods used for this research, followed by a section on relevant visual uncertainty representation approaches reported in literature. The article covers a dedicated section related to maps as a source of uncertainty. Further, our approach for visualisation of uncertainties on maps and with robustness indicators are presented. The final section formulates recommendations on visualisation of uncertainties and general recommendations related to maps to be used during the decision-engineering cycle in case of radiological releases.

\section{Method}

A multidisciplinary research approach has been applied and collaboration among different expertise and scientific domains has been established: nuclear emergency experts, radiation protection officers, risk communication experts, modellers, researchers of social sciences, risk communication and information processing. The following methods have been applied:

- non-systematic, thematic literature and document review to identify the relevant theoretical state-of-the art work in visualisation of uncertainties and to identify the main challenges in understanding the role of maps in recent nuclear or radiological emergencies (maps related to measurements of limited traces of radioactivity in the air from radiological releases ${ }^{106,103} \mathrm{Ru},{ }^{131} \mathrm{I}$ ) (Perko and Martell, 2019);

- modelling and cartographic solutions as examples to reflect with scientists and experts from various disciplines connected to radiation protection;

- non-participatory observation method applied in 11 national exercises in six countries throughout Europe, as well as one international exercise, with a total of 29 observation points, to assess understanding and interpretation levels of radiological maps used at the observed exercises;
- workshops and guided discussions in order to test the proposed maps and robustness indicators and collect comments (see Tab. 1).

Maps used for decision support in nuclear emergencies in general contain geographic information augmented with additional data such as dose rates and sheltering areas created by simulation software. The simulations are mainly based on several parameters such as weather prognosis, topology, source term and land use. Weather and source term are the main drivers of uncertainty in the assessment of a nuclear emergency (Leadbetter et al., 2018).

Source term uncertainty can be defined by a set of potential instances, e.g., worst, expected, and best estimate. Similarly, the weather uncertainty is defined by a set of different prognoses each with certain likelihood. By combining both of them, an ensemble of scenarios is created and evaluated, resulting in a set of maps for each possible map type like sheltering area.

At workshops and discussions five different cartographic solutions were presented as examples of a visual aid in case of a nuclear emergency. The examples of maps depicted a hypothetical but realistic case of a nuclear accident, with expected fallout of radioactivity (Fig. 1).

Table 1 summarises the feedback collection methods used to test the maps and the tools developed. For a comprehensive discussion on the stakeholders' engagement process and methods used, we refer the reader to Perko et al. (2019a, 2019b).

\section{Visual uncertainty representation approaches in literature}

Bertin presented eight visual elements that are relevant to represent a concept visually: position, size, shape, orientation, value (lightness), colour hue, orientation, texture (Bertin, 1983). Bertin defines the variable as selective if it is able to immediately catch the attention of the viewer over the entire plan without considering individual marks sequentially. 

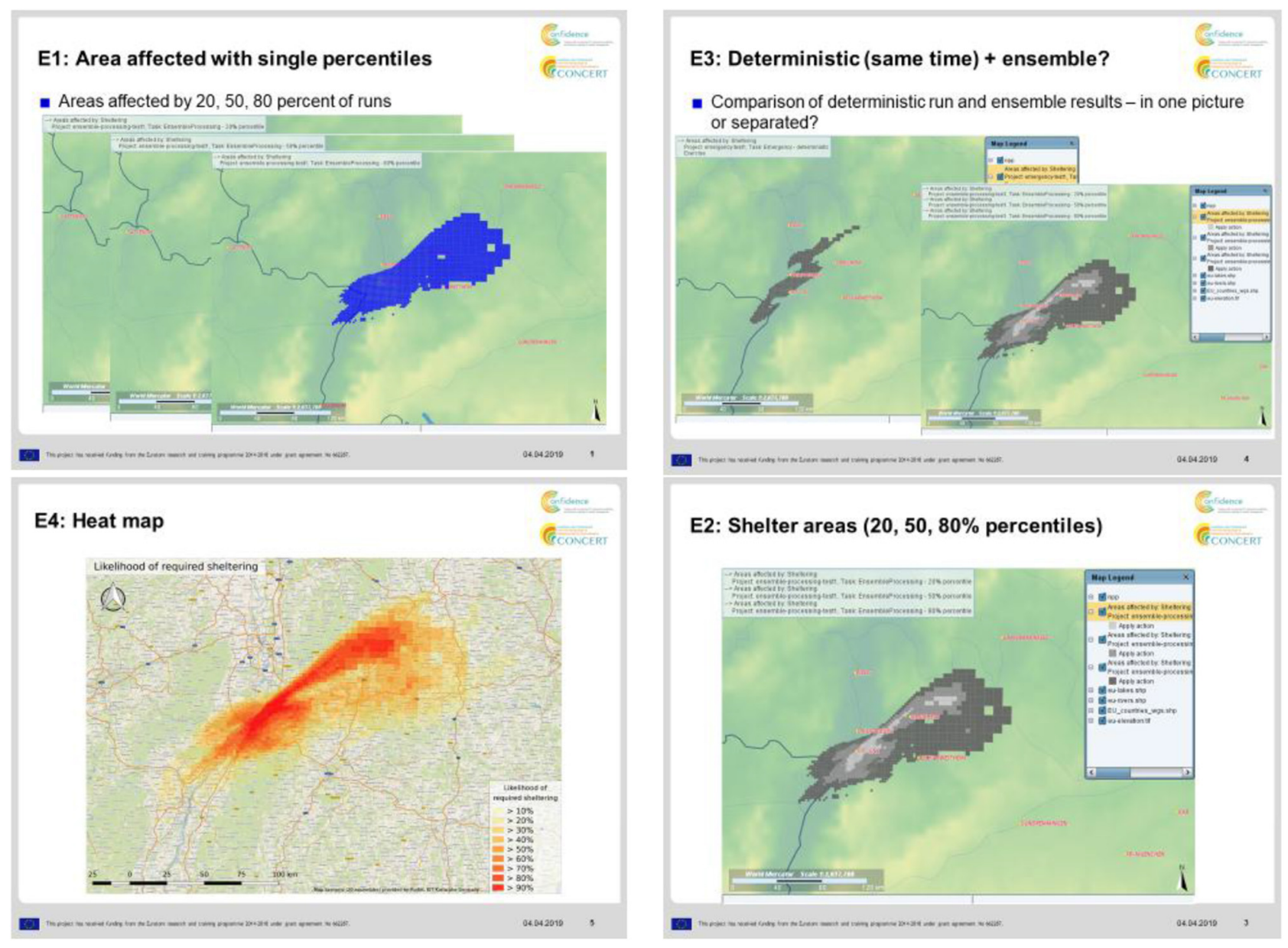

Fig. 1. Set of maps exceeding sheltering thresholds discussed with the participants in different countries (Source: KIT 2019). From top left to bottom right: 3 maps displaying percentiles likelihood, deterministic map vs. greyscale encoded binned likelihood, single colourful encoded likelihood, single percentile encode likelihood.

Healey defined visual representation based on attentive and pre-attentive processing, where the pre-attentive basic visual properties are detected immediately by low level visual system and do not require high cognitive overload (Healey et al., 1996).

Tufte identified an effective approach to visualize a concept as a way to give the viewer the greatest number of ideas in the shortest time (Tufte, 2001). Tufte identifies six principles in order to promote effective pre-attentive processing in the analyst/viewer's visual system. The five main principles could be summarized as follows: 1) avoid data distortion, 2) support data comparison whenever possible, 3) reveal multiple levels of detail in the data, 4) integrate statistical markers, and 5) integrate text description into the visual representation.

Ware extended the concepts presented by Tufte through identifying three principles relevant to our work, similar to Bertin's work. He referred to pre-attentive processing as one of the important aspects in developing effective visualization where marks can be used. Further, he stressed the need of inclusion of viewers with colour blindness and also mentioned that the usage of words is effective to present conditions (Ware, 2012).
Some of the early experimental work to visualize uncertainty for map-based measurements was carried out by Goodchild. The main findings of the work identified colour lightness ("value") as an ineffective method for encoding uncertainty on bivariate choropleth maps (Schweizer and Goodchild, 1992). In other words dark and light colours for encoding uncertainty on maps were not useful in conveying the meaning of uncertainty without prior training. Thus, decision makers do not perceive lightness and darkness as independent without briefing. As a result, decision makers in emergency planning should be briefed about the meaning of darker or lighter hues in order to result in better understanding of uncertainty and be able to trust the meaning of the uncertainty representation.

Evans developed an experiment to identify the effectiveness of the different options to represent uncertainty through dynamic display of spatial data reliability. Four options were compared: 1) static separate maps, 2) static integrated maps, 3) animated maps, 4) interactive maps (Evans, 1997). The work concluded that the static integrated display was the most effective to represent uncertainty for decision makers since it was less confusing. As a result, it is advised to integrate the data maps and uncertainty maps for decision makers to 
represent uncertainty according to Evans experiment. While data and uncertainty integration could provide an effective way to represent and handle uncertainty, resorting to interactive maps offers an opportunity to provide more information on demand in order to justify or motivate a decision.

Leitner and Buttenfield developed a guide for encoding uncertainty using a variety of attributes including saturation. The work concluded that visualization of information uncertainty/certainty significantly increases the number of correct responses for making decisions (Leitner and Buttenfield, 2000). Credible and certain data was encoded with lighter value or finer texture. Further, no difference in time for difficult or complex tasks was observed as a result of including uncertainty information.

Edwards and Nelson developed four ways to encode data quality and uncertainty in static graduated circle maps. The methods compared included textual and uncertainty indicators (color, value and texture). A graduated circle map to limit the number of levels of certainty or uncertainty was used which resulted in a significantly better display for the uncertainty according to the findings. The graduated circle map was compared as separate display and integrated where the display (uncertainty and data) yielded the best results (Edwards and Nelson, 2001). The reason behind that is the separate display offers more work for the user both perceptually and cognitively to construct the knowledge representation and process it. As a result, the integrated visualisation is less cognitively involving and demanding. Thus, to present information to radiological decision makers and emergency decision makers it is important to have a view where both data and uncertainty meta data are combined in one display.

While inter-comparisons with different ensembles can be an important part of building sound situational awareness for an emergency it might not lower the uncertainties perceived. As a result, careful selection of the model ensembles is necessary to present a balanced view on the range of uncertainties involved. Using reference areas has been an important recommendation by the IAEA through Response and Assistance Network (RANET, 2020). It is yet a challenging task to represent uncertainty without introducing further types or levels of uncertainty.

\section{Maps as a source of uncertainty recognized during exercises and radiological events}

Perko and Martell concluded that radiological maps are of great use in visualizing the affected environment since they reduce uncertainties and miscommunication (Perko and Martell, 2019); thus, offer an effective representation tool in emergency management. Unfortunately, radiological maps analysed in the context of this research were sometimes a source of uncertainty, misinterpretation and caused communication issues. The preliminary findings were inconsistent with our past experience which required further investigation.

\subsection{Findings based on user feedback}

There were many uncertainties on how to interpret radiological dispersion maps observed during emergency exercises. The following sentences taken from the observation notes reflect these uncertainties: "The picture (plume) used for the exercise has been misunderstood by some members. Although we use this type of maps, this is not OK. There is a need to use better maps that guarantees that these maps are correctly understood"; "Our decision makers don't understand such scientific maps. Decision makers need other maps."; "The most important information is missing on this map: intervention doses (it is not clear from the picture whether is this an ongoing release or prognoses), is it controlled release and what is duration of the release."; "The expert group sends by email to decision makers the maps and doses: they received back a lot of questions by decision makers-more clarity in the maps is asked."; "Misunderstanding regarding the release and the map: How to understand the (below) $6 \mathrm{~km}$ ?"; "Communication cell: Maps are discussed, however it is not easy for public information officers to understand what they depict."; "Measurement cell: "Coloured maps produced by JRODOS and HYSPLITFormat of the results have not been pre-defined (explanatory legends were missing)"; "PR asked for a copy of the maps and the measurements. A decision has been made to publish maps showing the plume. PR asked who is going to prepare explanatory notes about the maps and what extent of uncertainty we have regarding the data." (Perko et al., 2019b).

\subsection{Summary of gaps identified}

The following issues in visualisation on maps have been identified:

- maps lacking contextual information (e.g., on-going release or predicted release; missing legend);

- non-unified and diverse measurement units were used to describe the same attribute (e.g., $\mu \mathrm{Sv} / \mathrm{h}$ and $\mathrm{mR} / \mathrm{h}$ );

- diversity of colors has been used unrelated to the meaning of the colour (e.g., blue for the extremely low release, below legal norms);

- zones for protective actions indicated using country borders;

- scientific uncertainties not presented (e.g., related to the release time, meteorological conditions), low doses presented in many different ways (e.g., white colour, blue colour and units), no indication of health impact.

While gaps and issues in maps can limit their usefulness, they can be used as an effective means to build situational awareness and effectively represent uncertainty when proper design and usage guidelines are followed. In section 5, we propose recommendations addressing the different issues that showed up during the early versions of tools while testing.

\section{Proposed uncertainty visualisation guidelines for maps}

In order to support decision makers to process complex details while reducing heavily cognitive consuming details, we list below the design guidelines for the development of visual uncertainty representation tools (maps and robustness indicators) based on our literature review and experiments: 
Table 2. Indicator system based on five colours.

\begin{tabular}{|c|c|c|c|c|c|}
\hline Endpoint & $\begin{array}{l}\text { Early phase } \\
\text { (pre-release } \\
\text { and release) }\end{array}$ & $\begin{array}{l}\text { Early phase } \\
\text { based on } \\
\text { ensemble } \\
\text { modelling* }\end{array}$ & $\begin{array}{l}\text { Early phase } \\
\text { based on data } \\
\text { assimilation } \\
\text { (food and source } \\
\text { term) ** }\end{array}$ & Transition phase & $\begin{array}{l}\text { Long-term } \\
\text { recovery } \\
\text { phase }\end{array}$ \\
\hline Dose maps & red & yellow & red-yellow & yellow & green \\
\hline Dose rate maps & red & yellow & red-yellow & yellow & green \\
\hline $\begin{array}{l}\text { Countermeasure } \\
\text { areas }\end{array}$ & red-yellow & yellow & red-yellow & yellow & green \\
\hline $\begin{array}{l}\text { Plume arrival } \\
\text { time }\end{array}$ & red-yellow & yellow & n.a. & n.a. & n.a. \\
\hline $\begin{array}{l}\text { Concentration in } \\
\text { feed and } \\
\text { foodstuffs }\end{array}$ & red & yellow & yellow-green & yellow & green \\
\hline $\begin{array}{l}\text { Concentration in } \\
\text { rivers from run- } \\
\text { off }\end{array}$ & red & n.a. & n.a. & yellow & n.a. \\
\hline $\begin{array}{l}\text { Concentration in } \\
\text { rivers from } \\
\text { direct release }\end{array}$ & red-yellow & n.a. & n.a. & yellow & yellow \\
\hline $\begin{array}{l}\text { Concentration in } \\
\text { lakes and } \\
\text { reservoirs }\end{array}$ & red & n.a. & n.a. & yellow & yellow \\
\hline $\begin{array}{l}\text { Concentration in } \\
\text { marine food } \\
\text { products }\end{array}$ & red & n.a. & n.a. & yellow & yellow \\
\hline $\begin{array}{l}\text { Inhabited area } \\
\text { countermeasures }\end{array}$ & red & yellow & yellow & yellow & green \\
\hline $\begin{array}{l}\text { Food } \\
\text { countermeasures }\end{array}$ & red & yellow & yellow & yellow & green \\
\hline
\end{tabular}

*: indicates that performance should be increased if ensembles are very close; **: as long as release is on-going assimilation of source term is uncertain.

- Promote selective perception of the eye and emphasize the important regions and points;

- Support retrievability to provide explanation/reasoning behind the recommendation;

- Identify actionable recommendations in different uncertainty levels; whenever possible opt for robust adaptive strategies;

- Address colour blindness or low illumination conditions;

- Fuse different uncertainty magnitudes in one uncertainty index (e.g., source and measurements);

- Limit the number of uncertainty levels; keep them from 2 to 5 levels maximum; less levels are easier to reason with and make decisions based on them;

- Limit the introduction of new uncertainty in the visualization process.;

- Plan for training or briefing the decision makers through focus groups and group exercises or concise tutorials before usage of the uncertainty indicators;

- Express conditions and preconditions textually in the design and avoid representing them visually.

\section{Proposed robustness indicators for the uncertainty level of data presented at a map}

The purpose of the robustness indicators is to tell end-users if a result is appropriate for decision making indicating the range of uncertainty/quality linked with it. For testing the approach in the panels, a prototype was realised in JRODOS. Assuming that the source term and the weather data have different classification rules, there is a need to find a classification of results that is based on the two input parameters. A colour code with five colours is proposed to indicate the level of uncertainty (Tab. 2) and the letters (see Fig. 2) in order to make the robustness indicator functional for end-users with deficiencies of colour vision or dyschromatopsia.

\section{Recommendations related to visualisation of uncertainties through maps}

The recommendations related to visualisation of uncertainties through maps are following: 


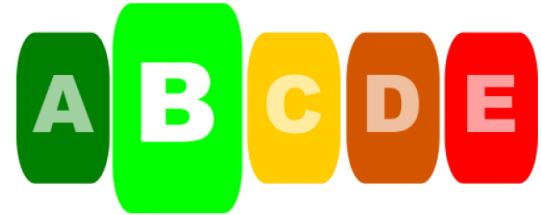

Fig. 2. Selected colour scheme as robustness indicator based on the French Nutri-score scheme (Julia et al., 2018).

- In order to aid the decision-making on the various countermeasures, a range of additional information should be available on maps besides the radiological situation (e.g., cities, population size, roads, drinking water reservoirs, evacuation routes etc.). However, since it is also important to keep the maps simple, interactive interfaces should be used where different layers with additional information could be turned on and off when needed;

- The design of the maps should be carefully thought through to make sure that it represents information in the most comprehensive way. The choice of colour coding should take into account existing codes that are already in use, the way different colours are perceived by people (e.g., red=danger); it should have enough contrast with the base map and be visible for people with disabilities;

- Maps should include informative legends with supplementary information on uncertainty among other things;

- It is extremely hard for people who are not used to maps and have not received any training to start using them in an emergency situation. Therefore, appropriate training and exercises for the use of maps are needed for the various stakeholders that will need to be involved in the decisionmaking;

- Where possible, uncertainty should be indicated on the maps and there are several solutions to how these could be presented, for example by varying brightness of colours, level of transparency and colour saturation, using glyphs and error bars. However, this representation will have to be situation-specific, since findings showed that even the expert participants were unable to articulate what their preferred uncertainty visualization methods were because they were convinced that this strongly depends on the task;

- Providing actionable recommendations is an important step towards increasing the value from the uncertainty representation whenever possible and applicable. When actionable recommendations are coupled with division of the map into zones and regions that offers better support for the decision maker and increases the trust in the uncertainty representation and communication;

- Support for examining patterns and distributions in the map is useful in addition to magnitudes to identify robust decisions;

- Emphasizing points and regions that might have a specific level of uncertainty helps focus attention of the decision makers to regions that the experts need to pay extra attention to the policies devised.

\section{Conclusions}

The work presented aims to identify effective approaches to visually represent uncertainty in the context of emergency planning with an emphasis on nuclear emergencies. Two tools were designed, developed and tested. The first was a map based visual representation while the second was a robustness indicator for the available information. A multidisciplinary approach was developed to identify and understand interpretation mechanisms of uncertainties presented on maps and robustness indicators by the different stakeholders. We identified the optimal approaches for application design and usage of uncertainty representation and communication. Further, we shared our best practices for relying on tools. Additional research work needs to be carried out in the future to further investigate potential causes for misinterpretation; the areas of work should target format, design, data and uncertainty. The future work should focus on interdisciplinary approaches from the fields of human computer interaction and social sciences. Future work should integrate theory based and empirical supported development of tools for visualization of uncertainties in particular, uncertainties during a radiological release.

Acknowledgements. The authors would like to extend their appreciation and acknowledge the effort of T.Duranova (VUJE), R. Sala (CIEMAT), V. Tafili (EEAE), Y. Tomkiv (NMBU), D. Oughton (NMBU), for facilitating testing the tools developed and compiling national reports on the tests, C. Benighaus for helping develop the protocol for testing the tools. The work described in this paper was conducted within the CONFIDENCE project which was part of the CONCERT project. This project has received funding from the Euratom research and training programme 2014-2018 under grant agreement No. 662287.

Disclaimer (Art. 29.5 GA). This publication reflects only the author's view. Responsibility for the information and views expressed therein lies entirely with the authors. The European Commission is not responsible for any use that may be made of the information it contains.

\section{References}

Bertin J. 1983. Semiology of graphics. University of Wisconsin Press. Edwards LD, Nelson ES. 2001. Visualizing data certainty: A case study using graduated circle maps. Cartogr. Perspect. 38: 19-26.

Evans BJ. 1997. Dynamic display of spatial data-reliability: does it benefit the map user? Comp. Geosci. 24(1): 1-14.

Healey CG, Booth KS, Enns JT. 1996. High-speed visual estimation using pre-attentive processing. ACM Trans. Comp.-Human Interact. (TOCHI) 3(2): 107-135.

Hunter GJ, Goodchild M. 1993. Managing uncertainty in spatial databases: putting theory into practice. URISA J. 5: 55-62.

Julia C, Etilé F, Hercberg S. 2018. Front-of-pack nutri-score labelling in France: an evidence-based policy. Lancet Public Health 3(4): e164.

Leadbetter S, Andronopoulos S, Bedwell P, Geertsema G, Jones A, Korsakissok I, Tomas J, De Vries H. 2018. Guidelines ranking uncertainties for atmospheric dispersion, D9.1.1 Using ensemble meteorological forecasts to represent meteorological uncertainty 
in dispersion models. CONCERT Deliverable D9.1. Available from https://concert-h2020.eu/en/Publications.

Leitner M, Buttenfield BP. 2000. Guidelines for the display of attribute certainty. Cartogr. Geogr. Inform. Sci. 27(1): 3-14.

Marignac Y, Hazemann J, Baudé S. 2016. Managing the complexity of societal needs in a nuclear emergency situation: towards further experts' collaboration for the "enlightened protection" of populations. Radioprotection 51(HS2): S159-S161.

Pang A, Wittenbrink C, Lodha S. 1997. Approaches to uncertainty visualization. Visual Comp. 13: 370-390.

Perko T, Martell M. 2019. Study on good practices in implementing the requirements on public information in the event of an emergency, under the Euratom Basic Safety Standards Directive and Nuclear Safety Directive-Final report. Luxembourg, $488 \mathrm{p}$.

Perko T, Benighaus L, Tafili V, Oughton DH, Tomkiv Y, Sala R, Germán S, López S, Oltra C, Duranova T, Raskob W, Müller T, Nishizawa M, Wolf HV, Thijssen P, Camps J, Turcanu C, Benighaus C, Moschner J, Renn O. 2019a. Guidelines on tools for communication of uncertainties. CONCERT Deliverable D9.29. Available from https://www.concert-h2020.eu/en/Publications.
Perko T, Tafili V, Sala R, Duranova T, Zeleznik N, Tomkiv Y, Hoti F, Turcanu C. 2019b. Report on observational study of emergency exercises: List of uncertainties. CONCERT Deliverable D9.28. Available from https://www.concert-h2020.eu/en/Publications.

RANET. 2020. IAEA Response and Assistance Network. Available from https://www.iaea.org/services/networks/ranet (Last accessed January 2020).

Raskob W, Schneider T, Gering F, Charron S, Zheleznyak M, Andronopoulos S, Heriard-Dubreuil G, Camps J. 2016. Editorial. Innovative integrative tools and platforms. Key results of the PREPARE European Project. Radioprotection 51(HS2).

Schweizer DM, Goodchild MF. 1992. Data quality and choropleth maps: An experiment with the use of color. Proc. GIS/LIS 2: 686-699.

Thomson J, Hetzler E, MacEachren AM, Gahegan M, Pavel M. 2005. A typology for visualizing uncertainty. Visualiz. Data Anal. 5669. International Society for Optics and Photonics.

Tufte ER. 2001. The visual display of quantitative information, Vol. 2. Cheshire, CT: Graphics Press.

Ware C. 2012. Information visualization: Perception for design. Elsevier.

Cite this article as: Nagy A, Perko T, Müller T, Raskob W, Benighaus L. 2020. Uncertainty visualization using maps for nuclear and radiological emergencies. Radioprotection 55(HS1): S197-S203 